\title{
Effect of depression on actual and perceived effects of reading rehabilitation for people with central vision loss
}

\author{
Patricia Grant, MS; ${ }^{1-3 *}$ William Seiple, PhD; ${ }^{1,3-4}$ Janet P. Szlyk, PhD ${ }^{1-3}$ \\ ${ }^{1}$ Research and Development, Jesse Brown Department of Veterans Affairs Medical Center, Chicago, IL; ${ }^{2}$ The Sandy \\ and Rick Forsythe Center for Comprehensive Vision Care at The Chicago Lighthouse for People Who are Blind or \\ Visually Impaired, Chicago, IL, ${ }^{3}$ Department of Ophthalmology and Visual Sciences, University of Illinois at Chicago, \\ Chicago, IL; ${ }^{4}$ Lighthouse International, New York, NY
}

\begin{abstract}
To investigate the relationship between depression and quantitative measures of visual function, we recruited 18 subjects with central scotomas from macular degeneration who were enrolled in a reading rehabilitation program. Psychological batteries and reading assessments were administered prior to rehabilitation; reading assessments and a measure of adaptation to vision loss were administered following rehabilitation. We investigated relationships between reported levels of depressive symptoms and reading and adaptation outcome measures by using Pearson product moment correlation analysis. Results revealed a significant relationship between depression levels and reading acuity difference scores $(r(16)=0.54, p=$ 0.02 ) and changes in adaptation to vision loss levels $(r(16)=0.62$, $p=0.01$ ), suggesting that those who reported greater depressive symptoms did not respond as well functionally to reading rehabilitation but reported greater improvement in levels of adaptation to vision loss following rehabilitation. Future research should focus on defining standard methods to assess and remediate depression as part of the rehabilitation process.
\end{abstract}

Clinical Trial Registration: ClinicalTrials.gov; NCT00125632, "Measuring Reading Rehabilitation Outcomes"; http://www. clinicaltrials.gov.

Key words: acquired vision loss, adaptation, age-related macular degeneration, central vision loss, depression, low vision, macular disease, outcomes, psychosocial factors, reading, vision rehabilitation, visual function.

\section{INTRODUCTION}

Approximately 3.4 million Americans over the age of 40 are currently affected by blindness or some form of visual impairment [1]. Age-related macular degeneration (AMD) ranks third among global causes of visual impairment and accounts for 8.7 percent of blindness cases. AMD remains the primary cause of visual deficiency in industrialized countries and is the leading cause of blindness in persons over age 65 [1]. By the year 2030, when the older population is expected to double, the number of people with blindness and other visual impairments will increase dramatically [2]. Therefore, strengthening current strategies of prevention and care to prepare for these demographic and epidemiologic trends is necessary.

Abbreviations: $\mathrm{AMD}=$ age-related macular degeneration, AVL = Adaptation to Age-Related Vision Loss Scale, CES-D = Center for Epidemiologic Studies Depression Scale, LogMAR = logarithm of the minimum angle of resolution, MMSE = MiniMental Status Examination, MNREAD = Minnesota Low Vision Reading Acuity Chart, PRL = preferred retinal locus, $\mathrm{SD}=$ standard deviation.

*Address all correspondence to Patricia Grant, MS; The Chicago Lighthouse for People Who Are Blind or Visually Impaired, Low Vision Research Laboratory, 1850 W. Roosevelt Rd, Chicago, IL 60616; 312-447-3233; fax: 312506-0111. Email: patricia.grant@chicagolighthouse.org DOI:10.1682/JRRD.2010.05.0080 
AMD is a progressive disease that creates severe difficulties in reading and in the performance of other daily activities. The unpredictable nature of the disease makes adaptation challenging and results in a psychological burden. Sudden vision loss causes changes in lifestyle that have a major effect on individuals, affecting both psychological functioning and quality of life. The inability to perform simple everyday tasks can lead to feelings of inadequacy, distress, and a loss of independence.

\section{Depression and Vision Loss}

The association between depression and visual impairment has been well documented [3-10]. In a recent study, Hayman et al. used depression inventories to determine that older adults with visual impairment report higher levels of depressive symptomatology than older adults without visual impairment [10]. It can be inferred that depression was directly related to the experience of vision loss, because the findings were independent of age, sex, living situation, ethnicity, number of medications taken, use of antidepressants, and visual acuity.

In addition, individuals with AMD exhibit levels of emotional distress regarding their vision loss that are comparable with those of individuals with other chronic debilitating disabilities such as arthritis and AIDS (acquired immune deficiency syndrome) [11]. Williams et al. determined that these feelings of distress can be directly attributed to a diagnosis of AMD, because they found no differences between patients with AMD only and patients with AMD in addition to other diseases [11]. Williams et al. also found that those with the most recent onset of AMD reported the highest levels of emotional distress. These findings are similar to those of Rees et al. [12]; in their study, 42 percent of the sample reported mild to severe depressive symptoms and the authors determined vision-specific distress to be the strongest predictor of depressive symptoms.

\section{Effect of Depression on Visual Function}

For individuals with central scotomas, using an eccentric retinal area for visual tasks is necessary, because visual tasks such as fixation and reading can no longer be performed effectively with the foveal area [13]. Reading is one of the most basic activities of daily living affected by vision loss; approximately 86 percent of patients who seek rehabilitation have problems with reading [14]. For this reason, and because it is so valu- able to communication, reading is often targeted as a goal of vision rehabilitation [15].

Very little research has been conducted on the relationship between vision rehabilitation and mental health outcomes among older adults with vision impairment. However, suggestions have been made that engaging in rehabilitative services has been associated with trends toward lower levels of depressive symptoms [3,5]. Rovner and Casten studied the interrelationships between disease severity, disability, and depression in 51 patients with AMD [7]. On the basis of in-home clinical interviews, 70 percent of patients reported activity loss caused by their compromised vision. Reading, driving, hobbies, and watching television were among the most frequently cited activities. Of these patients, 27 percent met the criteria for clinical depression. Correlations revealed significant relationships between depression and activity loss as well as disease severity and activity loss; however, a significant correlation between visual acuity and depression did not exist. Based on these findings, multinomial regression modeling was performed, and results revealed that jointly, visual acuity and activity loss accounted for 30 percent of the variance related to the level of affective distress. Rovner and Casten further concluded that disease severity affects the reported level of distress only to the extent that vision loss affects participation in valued activities.

Brody et al. [16] assessed the effects of participation in a self-management group intervention to determine whether moods, self-efficacy, and activity improved in patients with central vision loss as a result [7]. The results of this study showed that patients who received the intervention experienced significantly reduced distress, improved self-efficacy, and increased use of visual aids in comparison with the control patients who did not receive the intervention. This study demonstrates that a behavioral intervention can effectively improve psychological well being as well as visual function; however, standards for screening and treating depression in this group are still lacking.

To further advance the field's understanding of the individual components of rehabilitation, Horowitz et al. examined the effects of vision rehabilitation services (low-vision clinical services, skills training, and counseling) on depressive symptoms in 95 patients with low vision prior to the utilization of services and 2 years following [17]. Their study showed that 21 percent of the sample experienced consistent depression from baseline to followup, while 13 percent had remitted depression between 
baseline and follow-up. Horowitz et al. further analyzed the various components of the rehabilitation program to determine which services aided in reducing depression. They found that counseling services significantly contributed to a significant reduction in depressive symptoms. This is an important finding, because an investigation of 1,228 low-vision rehabilitation service entities in the United States revealed that 88 percent of available rehabilitation services solely offer optical aids with basic training, while psychological services and support groups are only offered 21 and 28 percent of the time, respectively [14].

\section{Current Study}

The purpose of our study was to explore the relationship between depression and reading rehabilitation outcomes. While self-reports of functional decline have been linked to depressive symptoms in people with vision impairment [18-19], we do not presently know how depression is related to quantitative outcome measures of visual function. We hypothesized that a significant relationship would exist between level of depression and rehabilitation outcomes, such that those who reported greater depressive symptoms would have poorer rehabilitation outcomes. We also hypothesized that a significant relationship would exist between level of depression and changes in adaptation to vision loss levels following rehabilitation, such that those with lower levels of depression would report greater levels of adaptation following rehabilitation.

\section{METHODS}

\section{Participants}

We recruited 18 individuals (8 female and 10 male) who had significant central vision loss caused by AMD from among participants enrolled in a reading rehabilitation program that aimed to improve reading speed in those with AMD [20]. Inclusion criteria comprised older individuals ( $\geq 50$ years) with a diagnosis of AMD. The visual acuity requirement comprised better than 20/400 Snellen equivalent and a documented central scotoma in the better-seeing eye. Exclusion criteria comprised those with a cognitive impairment (defined as having a MiniMental Status Examination [MMSE] [21] score of <24), serious health problems such as terminal illness, other major medical conditions, or a neurologic disorder. To complete the vision portion of the MMSE, participants used magnifiers and/or read enlarged text. None of the participants had a history of depression or were receiving any type of treatment for depression at the time of the study.

\section{Measures}

\section{Reading Performance}

We used the Minnesota Low Vision Reading Acuity Chart (MNREAD) [22] to measure reading acuity (logarithm of the minimum angle of resolution [LogMAR]), maximum reading speed (words per minute), and critical print size, which is the smallest print size the participant can read closest to their maximum reading rate. The sentence font sizes for these charts range from $1.3 \operatorname{logMAR}$ (20/400 Snellen equivalent) to -0.5 logMAR (20/6 Snellen equivalent). We instructed the participants to read the charts monocularly using their better-seeing eye, which was also the trained eye, at a standard reading distance of 16 in. $(40 \mathrm{~cm})$.

\section{Depression}

We used the Center for Epidemiologic Studies Depression Scale (CES-D) to assess the participants' levels of depression. The CES-D has well-established norms and established reliability and validity data. Correlations with other widely used depression inventories have also been reported [23]. We read statements to participants and asked them to determine how often during the previous week they had that experience. Scores can range from 0 to 60, with higher scores indicating greater feelings of depression. A cutoff score of 16 is typically indicative of "significant" depressive symptomatology. Examples of statements on this scale include "I was bothered by things that usually don't bother me" and "I felt lonely." The term "depression" in this study refers to depression as measured by the CES-D.

\section{Adaptation to Age-Related Vision Loss}

We used the Adaptation to Age-Related Vision Loss Scale (AVL) [24] to assess the participants' level of adaptation to their eye disease. This scale consists of items for which participants report their attitudes toward vision loss on a Likert scale ranging from 0 to 3 . Total scores can range from 0 to 72 , with higher scores indicating greater adaptive functioning. Validity and reliability for this measure have been demonstrated to have high internal consistency and significant correlations with commonly used scales [24]. Examples of items in this scale include "Because of my vision loss, I feel like I can never really 
do things for myself" and "A visually impaired person can never really be happy.”

\section{Procedures}

All participants enrolled in the study received a lowvision evaluation that included an optometrist prescription of reading glasses to be worn throughout the reading rehabilitation program. The program comprised one 2hour reading training session per week for 8 weeks. Each training session presented a series of exercises designed for participants to practice eye movements and use eccentric viewing techniques. Training began with identifying a parafoveal location, termed a preferred retinal locus (PRL), and advanced to reading single letters, words, and full sentences with the participant's PRL throughout the program. Reading was the primary outcome targeted. A trained optometrist administered all reading training. A previous publication from our laboratory provides more information regarding the development of this reading training protocol [20].

We administered reading performance and psychological assessments to each participant prior to their first rehabilitation session. On the last day of rehabilitation training, we assessed participants a second time using the reading and adaptation measures. Two different members of the research team administered the measures so that the participants' psychological status was not known at the time of reading assessments. Participants performed all reading training and reading assessments monocularly (using the trained eye).

\section{RESULTS}

Participants' ages ranged from 54 to 87 years (mean \pm standard deviation [SD]: $75.10 \pm 8.92$ years), with a mean visual acuity (measured by Lighthouse Visual Acuity charts) in their better eye of $0.82 \log$ MAR or 20/126 Snellen equivalent $(\mathrm{SD}=0.31)$ and mean Pelli-Robson contrast sensitivity of $0.99 \pm 0.42$. The racial breakdown of subjects was white $(n=15)$, black $(n=2)$, and Asian $(n=1)$. Scores on the CES-D ranged from 0 to 31 (median $=4.0$, standard error $=2.28$ ). We found no significant correlations between reported levels of depression and visual acuity $(r(16)=-0.15, p=0.57)$ or contrast sensitivity $(r(16)=0.08, p=0.76)$.

\section{Reading Outcome Measures}

We calculated difference scores (post- minus prerehabilitation training) for each measure of reading performance. Pearson product moment correlation analysis revealed a significant relationship between levels of depression and change in MNREAD $(r(16)=0.54, p=$ 0.02) (Figure 1). The correlation suggests that those participants who reported less depression experienced greater improvement in this measure following rehabilitation training. We found no significant correlations between levels of depression and the maximum reading speed and critical print size measures.

\section{Adaptation to Vision Loss}

We calculated difference scores (post- minus prerehabilitation training) for the AVL. Pearson product moment correlation analysis revealed a significant relationship between levels of depression and change in levels of adaptation to vision loss $(r(16)=0.62, p=0.01)$, indicating that those participants who reported greater depression at the start of rehabilitation reported a greater positive change in adaptation after rehabilitation (Figure 2).

\section{DISCUSSION}

\section{Relationship Between Depression and Reading Outcome Measures}

This study investigated the relationship between participants' perceived level of depression and changes in reading performance and levels of adaptation to vision loss following vision rehabilitation. An analysis of the

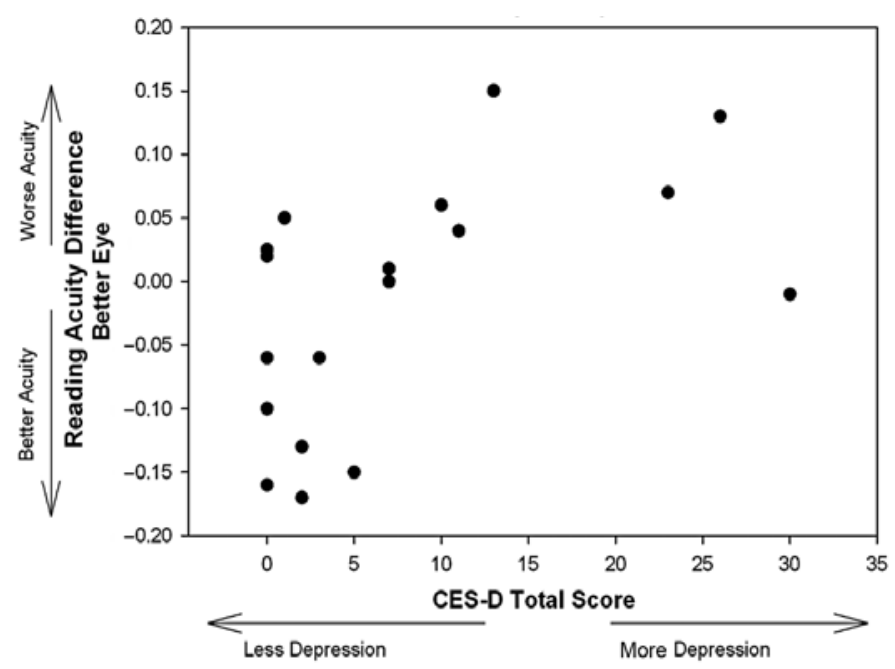

Figure 1.

Correlation between baseline Center for Epidemiologic Studies Depression Scale (CES-D) depression level and Minnesota Low Vision Reading Acuity Chart outcome measure (post minus baseline score). 


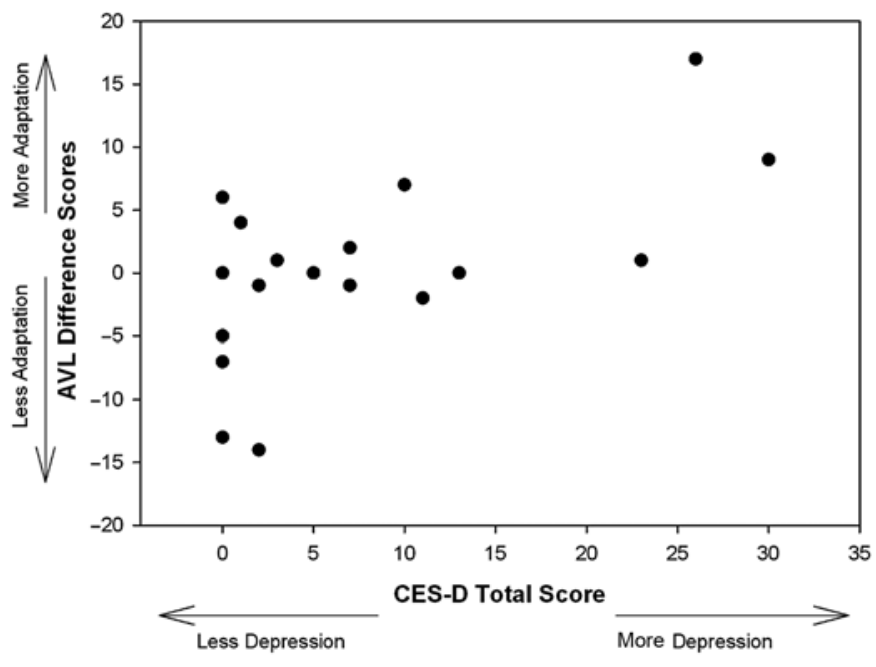

Figure 2.

Correlation between baseline Center for Epidemiologic Studies Depression Scale (CES-D) depression level and Adaptation to Age-Related Vision Loss Scale (AVL) outcome measure (post minus baseline score).

relationship between depressive levels and reading measures indicated that levels of depression can negatively affect intended reading rehabilitation outcomes. These findings are consistent with findings from Rovner et al. [18] and Iliffe et al. [19] showing that depression can prevent patients with low vision from achieving optimal vision rehabilitation outcomes.

Patients with AMD face a number of challenges regarding their eye disease and advancing vision loss, specifically from stress caused by loss of independence, the inability to perform everyday activities, and the risk of social isolation. Low-vision rehabilitation programs have been developed to help patients with low vision regain daily functioning abilities. The overall goal of these programs is to address physical adjustment of the patients, while psychosocial issues are rarely addressed. This is an area that should be considered, because as documented by this research, these issues can influence the way in which individuals adapt to their vision loss, as well as how they function.

Hayman et al. found that subjective perceptions of health were more important in explaining feelings of depression than the actual objective measures of visual function [10]. This article supports that finding, because reported depressive levels were independent of actual visual acuity measurements. Currently, no research has explored patient readiness for vision rehabilitation programs. Our research offers insight into the need to prop- erly assess participants prior to enrollment in a vision rehabilitation program to maximize the effectiveness of the program.

\section{Relationship Between Depression and Adaptation to Vision Loss}

A secondary purpose of this study was to examine the relationship between levels of depression and feelings of adaptation to vision loss. The results were interesting, because Figure 2 shows that those who reported more depressive symptoms at the study's onset reported a greater change in feelings of adaptation following rehabilitation. We did not administer a postrehabilitation depression measure in this study; therefore, it is unknown whether depressive symptoms declined as well. However, these findings are consistent with a study by Reinhardt [25], who reports that instrumental support was imperative to increasing levels of adaptation.

Vision rehabilitation services can encourage older adults with vision impairment. During training, the rehabilitation therapist works one-on-one with the patient. The components of rehabilitation can help restore activities that were once lost because of vision loss [8]. In addition, literature has shown that reading rehabilitation services have served as an independent predictor of decreased depressive symptoms in older adults with visual impairment $[8-9,15]$. However, more research is needed to determine which aspects of vision rehabilitation can best help relieve depressive symptoms.

\section{Limitations}

This sample size was sufficient to test the research questions proposed; however, a larger sample size is needed for more powerful analyses. In addition, only a small portion of the participants reported depression that fell into the significantly depressed level of the CES-D (total score $>16$ ). One reason for this may be that patient referral to a vision rehabilitation program is currently not standard care; therefore, those who did enroll in the program had to exhibit a level of proactive behavior by seeking out the program.

For this study, depression was represented by a selfreport depression scale. Those participants who did not feel comfortable reporting depressive symptoms may possibly have been misclassified. Also, we administered no psychological inventories at the end of the study. Therefore, the effect that participation in the rehabilitation program had on depressive symptoms is unknown. 


\section{CONCLUSIONS}

Individuals who experience vision loss late in life do not just lose visual function but also independence and social networks [26]. These experiences can lead to emotional distress and depression. While vision rehabilitation programs are designed to increase the level of functional ability by optimizing the remaining vision, this article demonstrates that psychological status may influence vision rehabilitation outcomes. This factor is often overlooked in the development of vision rehabilitation programs.

\section{ACKNOWLEDGMENTS}

\author{
Author Contributions: \\ Study concept and design: P. Grant, W. Seiple, J. P. Szlyk. \\ Acquisition of data: P. Grant. \\ Analysis and interpretation of data: P. Grant, W. Seiple. \\ Drafting of manuscript: P. Grant, W. Seiple, J. P. Szlyk. \\ Critical revision of manuscript for important intellectual content: \\ P. Grant, W. Seiple, J. P. Szlyk. \\ Statistical analysis: P. Grant, W. Seiple. \\ Obtained funding: W. Seiple, J. P. Szlyk. \\ Administrative, technical, or material support: P. Grant. \\ Study supervision: J. P. Szlyk.
}

Financial Disclosures: The authors have declared that no competing interests exist.

Funding/Support: This material was based on work supported by the Department of Veterans Affairs, Office of Research and Development, Rehabilitation Research and Development Service Merit Review (grant O3877R to Dr. Szlyk).

Additional Contributions: The authors thank Grace Soong, OD, for administering the reading rehabilitation training.

Institutional Review: We obtained informed consent from all study participants in adherence to the Declaration of Helsinki.

Participant Follow-Up: The authors do not plan to inform participants of the publication of this study. However, participants have been encouraged to check the study Web site for updated publications.

\section{REFERENCES}

1. Friedman DS, Congdon N, Kempen J, Tielsch JM. Vision problems in the U.S.: Prevalence of adult vision impairment and age-related eye disease in America. Bethesda (MD): National Eye Institute; 2002.

2. Kempen JH, Mitchell P, Lee KE, Tielsch JM, Broman AT, Taylor HR, Ikram MK, Congdon NG, O’Colmain BJ; Eye Diseases Prevalence Research Group. The prevalence of refractive errors among adults in the United States, Western Europe, and Australia. Arch Ophthalmol. 2004;122(4):
495-505. [PMID: 15078666]

DOI:10.1001/archopht.122.4.495

3. Bernbaum M, Albert SG, Duckro PN. Psychosocial profiles in patients with visual impairment due to diabetic retinopathy. Diabetes Care. 1988;11(7):551-57. [PMID: 3203572] DOI:10.2337/diacare.11.7.551

4. Karlsson JS. Self reports of psychological distress in connection with various degrees of visual impairment. J Vis Impair Blind. 1998;92(7):483-90.

5. Bailey P, Dodds AG, Pearson A, Yates L. Psychological factors in acquired visual impairment: The development of a scale of adjustment. J Vis Impair Blind. 1991;85(7):306-10.

6. Szlyk JP, Becker JE, Fishman GA, Seiple W. Psychological profiles of patients with central vision loss. J Vis Impair Blind. 2000;94(12):781-86.

7. Rovner BW, Casten RJ. Activity loss and depression in age-related macular degeneration. Am J Geriatr Psychiatry. 2002;10(3):305-10. [PMID: 11994218]

8. Horowitz A, Reinhardt JP, Boerner K, Travis LA. The influence of health, social support quality and rehabilitation on depression among disabled elders. Aging Ment Health. 2003;7(5):342-50. [PMID: 1295803] DOI:10.1080/1360786031000150739

9. Bragg MW. Vision loss, depression and rehabilitation. Int Congr Ser. 2005;1282:40-41. DOI:10.1016/j.ics.2005.06.028

10. Hayman KJ, Kerse NM, La Grow SJ, Wouldes T, Robertson MC, Campbell AJ. Depression in older people: Visual impairment and subjective ratings of health. Optom Vis Sci. 2007;84(11):1024-30. [PMID: 18043421] DOI:10.1097/OPX.0b013e318157a6b1

11. Williams RA, Brody BL, Thomas RG, Kaplan RM, Brown SI. The psychosocial impact of macular degeneration. Arch Ophthalmol. 1998;116(4):514-20. [PMID: 9565052]

12. Rees G, Tee HW, Marella M, Fenwick E, Dirani M, Lamoureux EL. Vision-specific distress and depressive symptoms in people with vision impairment. Invest Opthalmol Vis Sci. 2010;51(6):2891-96. [PMID: 20164466] DOI:10.1167/iovs.09-5080

13. Schuchard RA, Naseer S, De Castro K. Characteristics of AMD patients with low vision receiving visual rehabilitation. J Rehabil Res Dev. 1999;36(4):294-302. [PMID: 10678452]

14. Owsley C, McGwin G Jr, Lee PP, Wasserman N, Searcey $\mathrm{K}$. Characteristics of low-vision rehabilitation services in the United States. Arch Ophthalmol. 2009;127(5):681-89. [PMID: 19433720] DOI:10.1001/archophthalmol.2009.55

15. American Academy of Ophthalmology: Refractive Errors Panel. Rehabilitation: The management of adult patients with low vision. San Francisco (CA): American Academy of Ophthalmology; 1994.

16. Brody BL, Williams RA, Thomas RG, Kaplan RM, Chu RM, Brown SI. Age-related macular degeneration: A randomized clinical trial of a self-management intervention. 
Ann Behav Med. 1999;21(4):322-29. [PMID: 10721440$]$ DOI:10.1007/BF02895965

17. Horowitz A, Reinhardt JP, Boerner K. The effect of rehabilitation on depression among visually disabled older adults. Aging Ment Health. 2005;9(6):563-70. [PMID: 16214704] DOI:10.1080/13607860500193500

18. Rovner BW, Casten RJ, Tasman WS. Effect of depression on vision function in age-related macular degeneration. Arch Ophthalmol. 2002;120(8):1041-44. [PMID: 12149057]

19. Iliffe S, Kharicha K, Harari D, Swift C, Gillmann G, Stuck A. Self-reported visual function in healthy older people in Britain: An exploratory study of associations with age, sex, depression, education and income. Fam Pract. 2005;22(6): 585-90. [PMID: 16055468]

DOI:10.1093/fampra/cmi067

20. Seiple W, Szlyk JP, McMahon T, Pulido J, Fishman GA. Eye-movement training for reading in patients with agerelated macular degeneration. Invest Ophthalmol Vis Sci. 2005;46(8):2886-96. [PMID: 16043863]

DOI:10.1167/iovs.04-1296

21. Folstein MF, Folstein SE, McHugh PR. "Mini-mental state." A practical method for grading the cognitive state of patients for the clinician. J Psychiatr Res. 1975;12(3):189-98. [PMID: 1202204] DOI:10.1016/0022-3956(75)90026-6

22. Mansfield JS, Ahn SJ, Legge GE, Luebker A. A new readingacuity chart for normal and low vision. In: Noninvasive assessment of the visual system technical digest. Washington (DC): Optical Society of America; 1993. p. 232-35.
23. Radloff LS. The CES-D Scale: A self report depression scale for research in the general population. Appl Psychol Meas. 1997;1(3):385-401.

DOI:10.1177/014662167700100306

24. Horowitz A, Reinhardt JP. Development of the adaptation to age-related vision loss scale. J Vis Impair Blind. 1998; 92(1):30-41.

25. Reinhardt J. Effects of positive and negative support received and provided on adaptation to chronic visual impairment. Appl Dev Sci. 2001;5(2):76-85.

DOI:10.1207/S1532480XADS0502 3

26. Davis C, Lovie-Kitchin J, Thompson B. Psychosocial adjustment to age-related macular degeneration. $\mathrm{J}$ Vis Impair Blind. 1995;89(1):16-27.

Submitted for publication May 4, 2010. Accepted in revised form March 25, 2011.

This article and any supplementary material should be cited as follows:

Grant P, Seiple W, Szlyk JP. Effect of depression on actual and perceived effects of reading rehabilitation for people with central vision loss. J Rehabil Res Dev. 2011;48(9):1101-8.

DOI:10.1682/JRRD.2010.05.0080

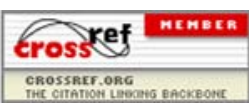


